\title{
Simulation of the HERMEs Lead Glass Calorimeter using a LUT
}

\author{
A. Vandenbroucke ${ }^{1} \quad$ C.A. Miller ${ }^{2}$ \\ On behalf of the HERMES Collaboration \\ ${ }^{1}$ Department of Subatomic and Radiation Physics \\ University of Gent,Belgium \\ ${ }^{2}$ TRIUMF \\ Vancouver, Canada
}

XIlth International Conference on Calorimetry in High Energy Physics, Chicago, June 2006 


\section{Outline}

(1) Introduction

- The HeRmes Experiment

- Design of the HERMES Lead-Glass Calorimeter

(2) Simulation of the Lead Glass Calorimeter

- Description of the Simulation

- Implementation of the Algorithm

(3) Results of the Simulation

- Different particles and observables 


\section{Outline}

(1) Introduction

- The HeRmes Experiment

- Design of the HERMES Lead-Glass Calorimeter

Simulation of the Lead Glass Calorimeter

- Description of the Simulation

- Implementation of the Algorithm

Results of the Simulation

- Different particles and observables 


\title{
The HeRmEs Experiment
}

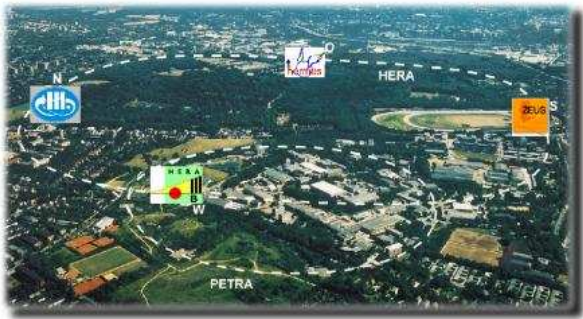

\author{
HERA \\ $\Rightarrow \quad$ MEasurement \\ of Spin
}

- Spin like Charge fundamental property

- Experiment at DESY Hamburg

- $27,5 \mathrm{GeV}$ longitudinally polarised $e^{ \pm}$from HERA accelerator

- Running since 1995 


\section{HeRMes Physics} A Very Short Overview

(1) Inclusive Physics:

$$
\begin{aligned}
& e+p \rightarrow e+X \\
& \Rightarrow x \cdot g_{1}\left(Q^{2}, x\right)
\end{aligned}
$$
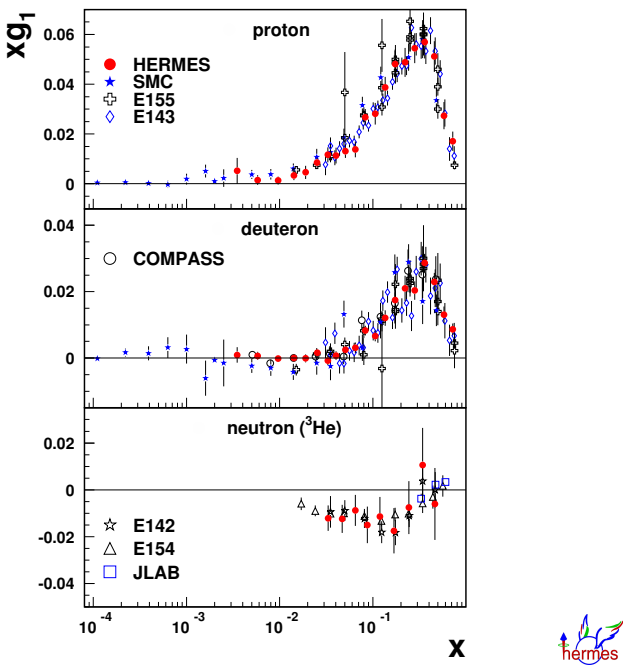


\section{HeRMes Physics}

\section{A Very Short Overview}

(1) Inclusive Physics:

$e+p \rightarrow e+X$

$\Rightarrow x \cdot g_{1}\left(Q^{2}, x\right)$

(2) Semi-Inclusive Physics: $e+p \rightarrow e+\pi^{ \pm} / K^{ \pm}+X$

$\Rightarrow x \cdot \Delta q\left(Q^{2}, x\right)$
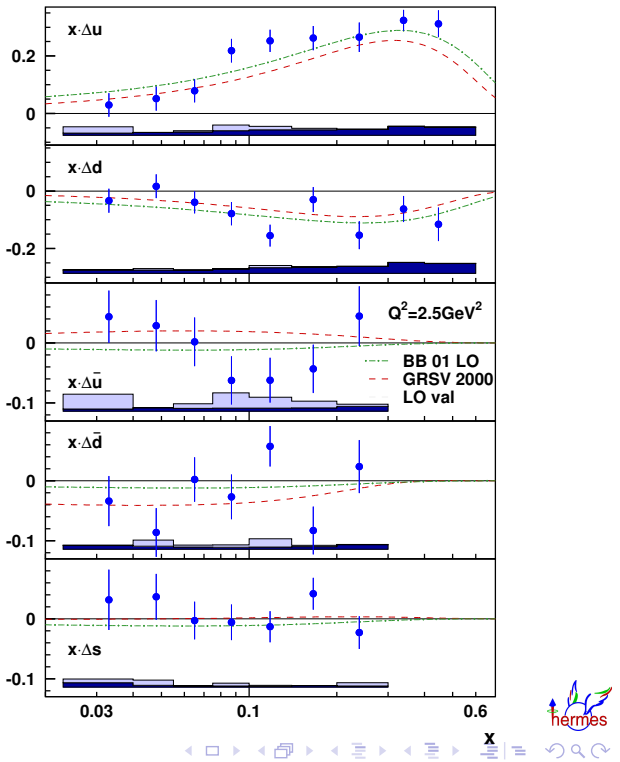


\section{HeRMes Physics}

A Very Short Overview

(1) Inclusive Physics:

$e+p \rightarrow e+X$

$\Rightarrow x \cdot g_{1}\left(Q^{2}, x\right)$

(2) Semi-Inclusive Physics: $e+p \rightarrow e+\pi^{ \pm} / K^{ \pm}+X$

$\Rightarrow x \cdot \Delta q\left(Q^{2}, x\right)$

(3) Exclusive Physics:

$\boldsymbol{e}+p \rightarrow \boldsymbol{e}+\gamma+p$

$\Rightarrow J^{q}\left(x, Q^{2}\right)$

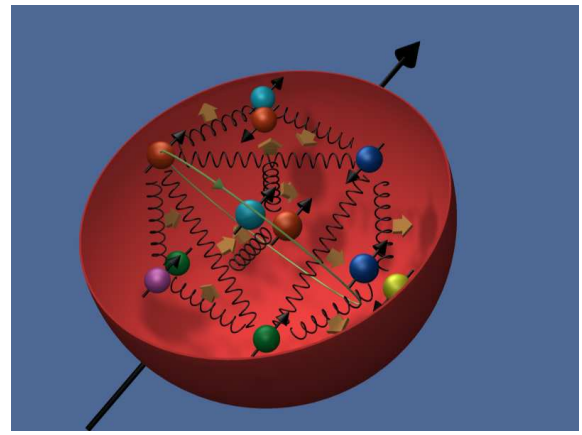

$$
\frac{1}{2}=\Delta \Sigma+L_{q}+J_{g}
$$




\section{The HERMES Spectrometer}

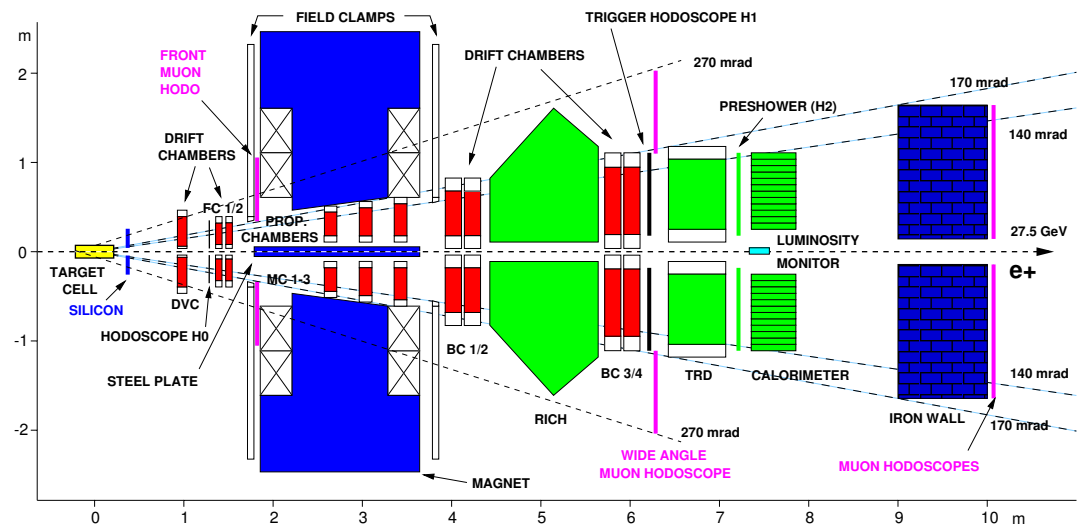

- Internal Polarized Gas Target

- Magnet Momentum measurement

- $\mathrm{RICH}$ to separate pion,kaon, proton

- Tracking Chambers $\Delta P / P \sim 2 \%$

Calorimeter

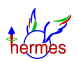

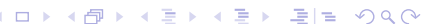




\section{The HERMES Spectrometer}

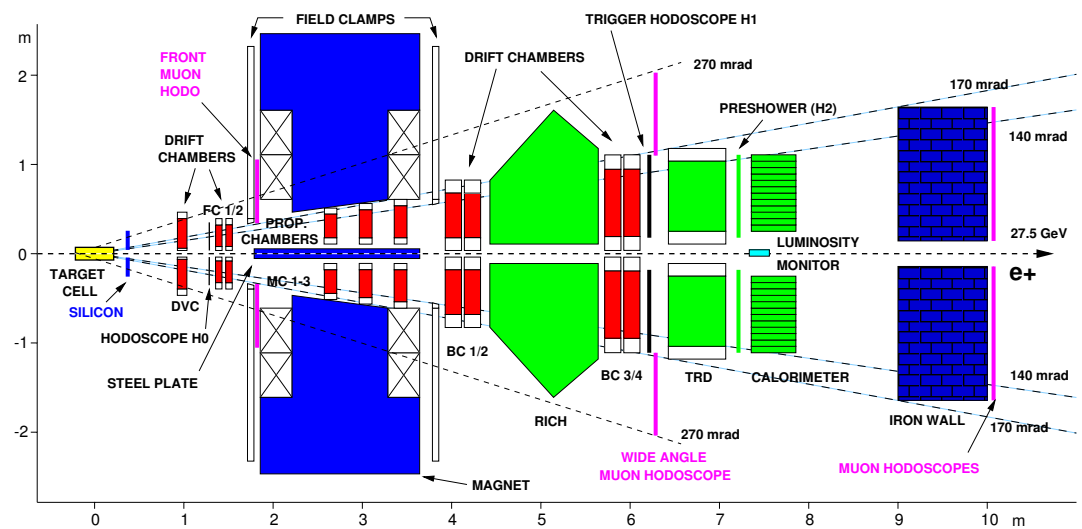

- Internal Polarized Gas Target

- Magnet Momentum measurement

- Tracking Chambers $\triangle P / P \sim 2 \%$

- Lepton/Hadron Separation with $\epsilon>99 \%$

- $\mathrm{RICH}$ to separate pion,kaon,proton

- Calorimeter 


\section{Outline}

(1) Introduction
- The HERMES Experiment
- Design of the Hermes Lead-Glass Calorimeter

\section{Simulation of the Lead Glass Calorimeter}

- Description of the Simulation

- Implementation of the Algorithm

Results of the Simulation

- Different particles and observables 


\section{Preshower - Calorimeter combination}

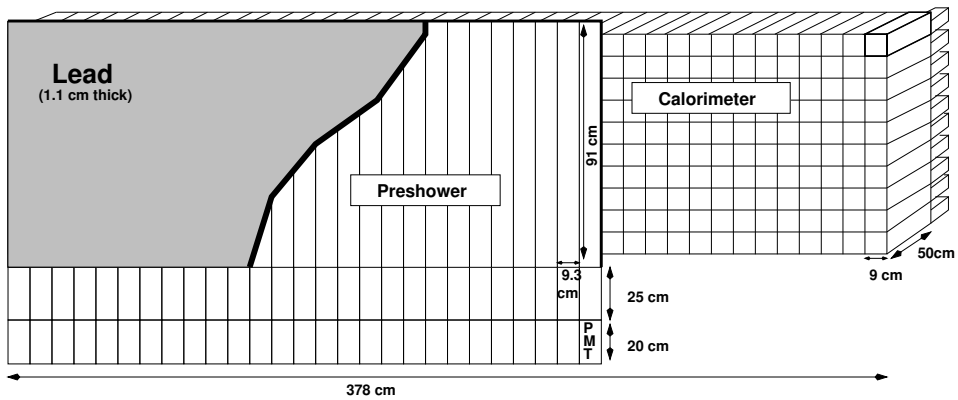

- Serves as a first level trigger

- Electron/Hadron Separation (preshower removes $\pi$ background)

- Identify $\pi^{0}$ through its decay in $2 \gamma^{\prime} s$

- Give a coarse position estimation 


\section{Design of the HERMES EM Calorimeter}

- Calorimeter was build by LNF,NIKHEF, and YPI.

- Build out of $2 \times 42 \times 10$ Lead Glass F101 blocks (Rad. Hard)

- Block Surface of $9 \times 9 \mathrm{~cm}$ ( $>90 \%$ of lateral shower profile )

- Block Length of 50 centimeter ( $18 \times$ Radiation length )

\begin{tabular}{|cl|}
\hline Chemical Composition F101 & weight \% \\
$\mathrm{PB}_{3} \mathrm{O}_{4}$ & 51.23 \\
$\mathrm{SiO}_{2}$ & 41.53 \\
$\mathrm{~K}_{2} \mathrm{O}$ & 7.0 \\
$\mathrm{Ce}$ & 0.2 \\
\hline \hline Radiation Length & $2.78 \mathrm{~cm}$ \\
Critical Energy & $17.97 \mathrm{MeV}$ \\
Refraction index & 1.65 \\
Molière Radius & $3.28 \mathrm{~cm}$ \\
\hline
\end{tabular}




\section{Outline}

\section{Introduction}

\section{- The HeRMES Experiment \\ - Design of the HeRMES Lead-Glass Calorimeter}

(2) Simulation of the Lead Glass Calorimeter

- Description of the Simulation

- Implementation of the Algorithm

Results of the Simulation

- Different particles and observables 


\section{Calorimeter in the HeRMEs Monte Carlo}

- New physics requires a better simulation

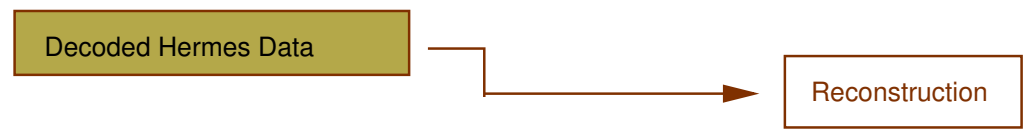




\section{Calorimeter in the HeRMEs Monte Carlo}

- New physics requires a better simulation
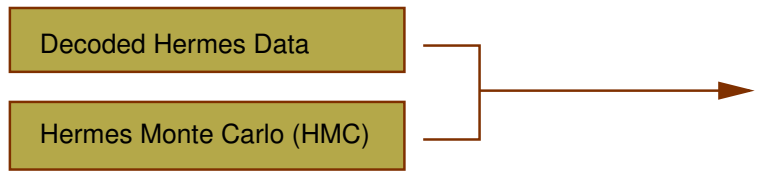

\section{Reconstruction}




\section{Calorimeter in the HERMEs Monte Carlo}

- New physics requires a better simulation
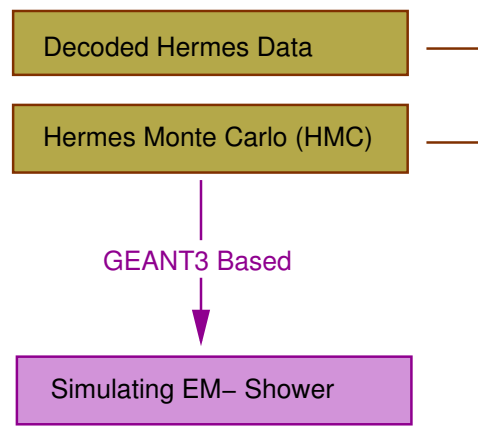

\section{Reconstruction}




\section{Calorimeter in the HERMEs Monte Carlo}

- New physics requires a better simulation

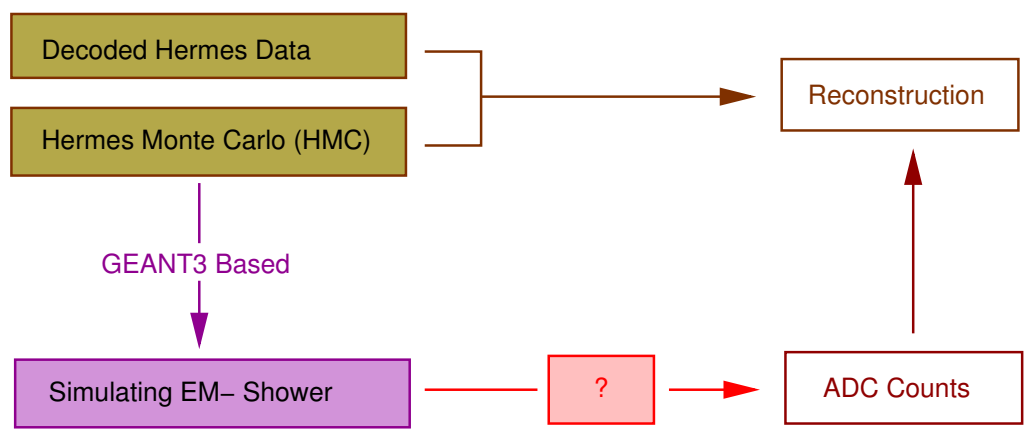




\section{Calorimeter in the HERMEs Monte Carlo}

- New physics requires a better simulation

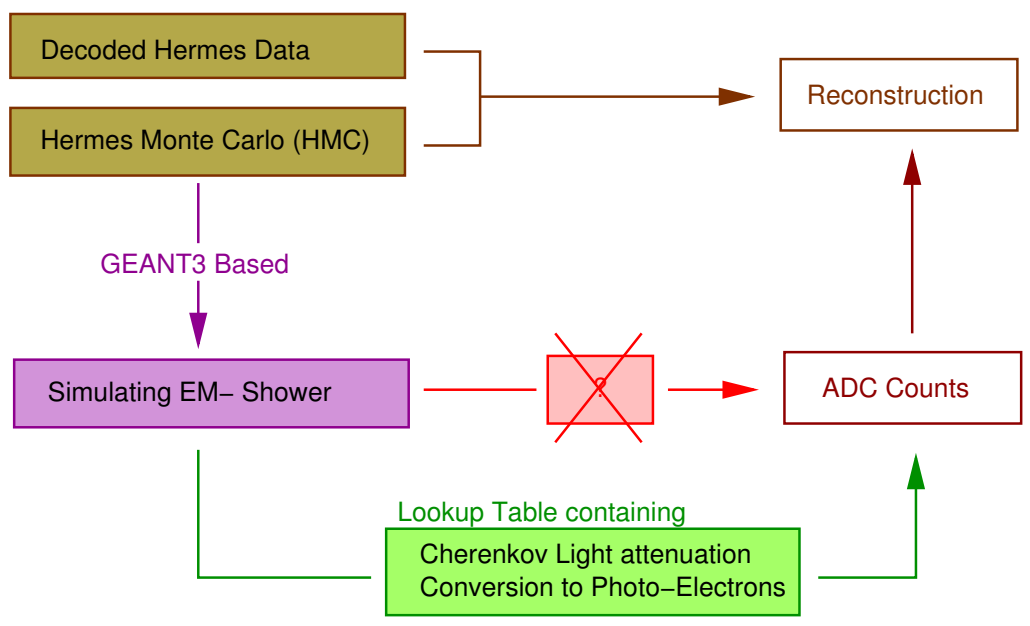




\section{Effects to the Cherenkov Light}

\section{From Cherenkov Light To Photo-Electron}

- Reflectivity of the foils

- Transparancy of the Lead

- Reflection at all surfaces (including glue)

- Quantum Efficiency PMT
Measurements at U. Hamburg

Measurements with spherical spectrophotometer

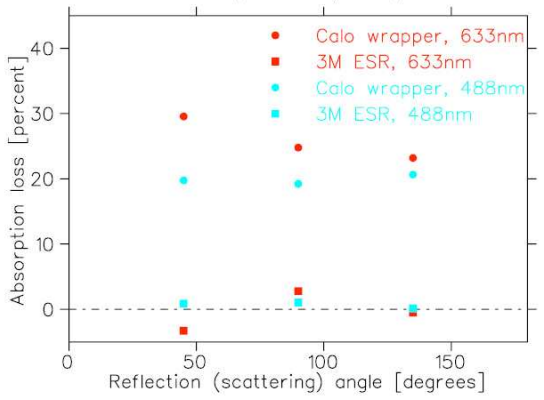




\section{Effects to the Cherenkov Light}

\section{From Cherenkov Light To Photo-Electron}

- Reflectivity of the foils

- Transparancy of the Lead Glass

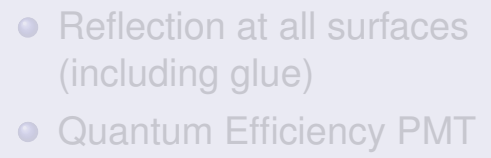

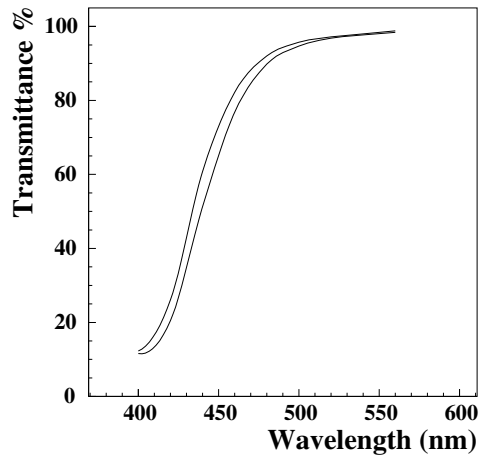




\section{Effects to the Cherenkov Light}

\section{From Cherenkov Light To Photo-Electron}

- Reflectivity of the foils

- Transparancy of the Lead Glass

- Reflection at all surfaces (including glue)

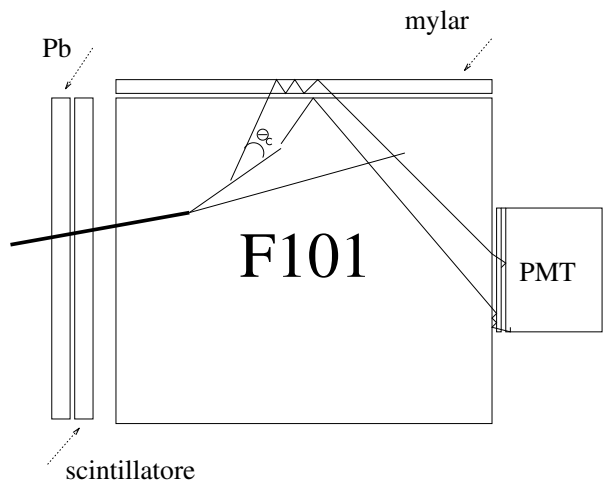




\section{Effects to the Cherenkov Light}

XP3461 Quantum Efficiency

\section{From Cherenkov Light To Photo-Electron}

- Reflectivity of the foils

- Transparancy of the Lead Glass

- Reflection at all surfaces (including glue)

- Quantum Efficiency PMT

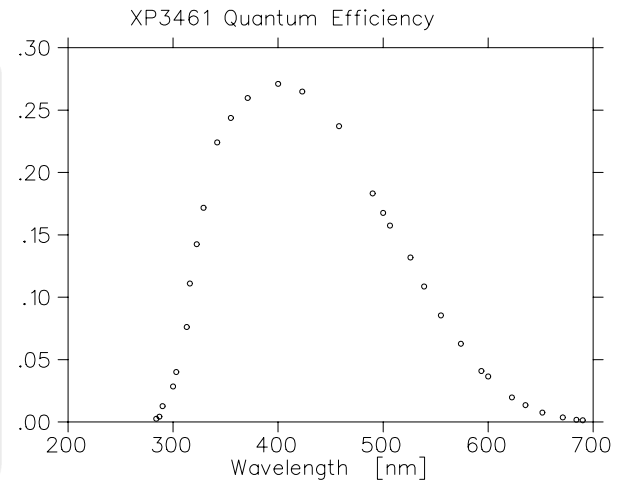


From Cherenkov Light To Photo-Electron

- Reflectivity of the foils

- Transparancy of the Lead Glass

- Reflection at all surfaces (including glue)

- Quantum Efficiency PMT
Stand Alone (G4 Based) Monte Carlo

\author{
$\Downarrow$ \\ Lookup Table
}




\section{Determining the LUT dimensionality}

- Cherenkov angle $\cos \left(\theta_{C}\right)=\frac{1}{\beta \cdot n}$ is velocity dependent

- Cherenkov radiation forward and backward

- Amount of Reflections dependent on the $(x, y)$ position
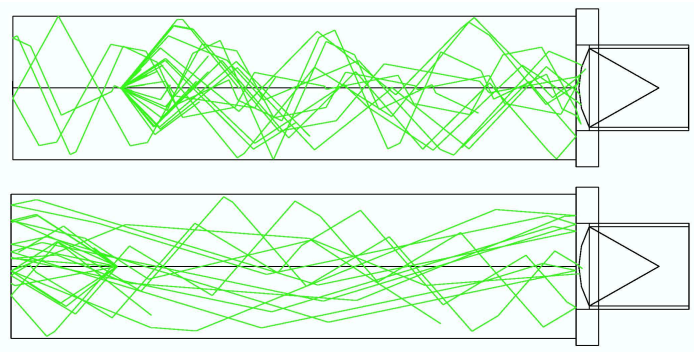


\section{Determining the LUT dimensionality}

- Cherenkov angle $\cos \left(\theta_{C}\right)=\frac{1}{\beta \cdot n}$ is velocity dependent

- Cherenkov radiation forward and backward

- Amount of Reflections dependent on the $(x, y)$ position
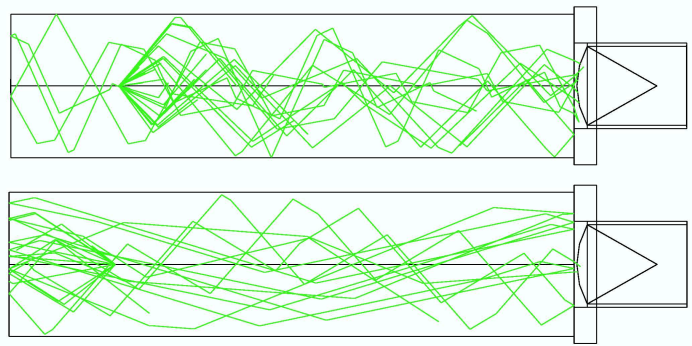

$\Rightarrow 6$ Dimensional LUT CONTAINING PE $\left(x, y, z, p_{\theta}, p_{\phi}, \beta\right)$ 


\section{Outline}

\section{Introduction}

- The HERMES Experiment

- Design of the HERMES Lead-Glass Calorimeter

(2) Simulation of the Lead Glass Calorimeter

- Description of the Simulation

- Implementation of the Algorithm

Results of the Simulation

- Different particles and observables 


\section{Interpolating the Table}

- Having a $10 \times 10 \times 16 \times 40 \times 10 \times 16$ grid, we want the right number of PE (per $\mathrm{mm} \mathrm{e}^{-}$track length) for any $\left(x_{1} \cdots x_{6}\right) \in$ the grid

- The most simple case is to extend a linear algorithm to 6 dimensions, yielding $2^{6}-1$ interpolations.

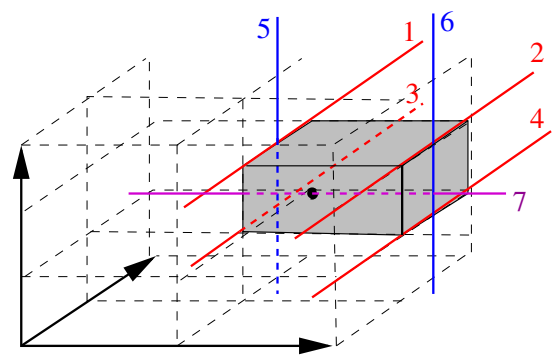

- The CERnLIB FINT algorithm based on linear interpolation has been extended to 6 dimensions. 


\section{Interpolating the Table II}

- Another method is the simplex method. Supposedly the fastest method possible[1].

- A hypercube around the point $x_{i}$ is normalized to the $n$-dimensional unit cube

- The unit cube then can be further divided into simplexes. An $n$-simplex is a $n$-dimensional analogue of a triangle, eg a tetrahedron.
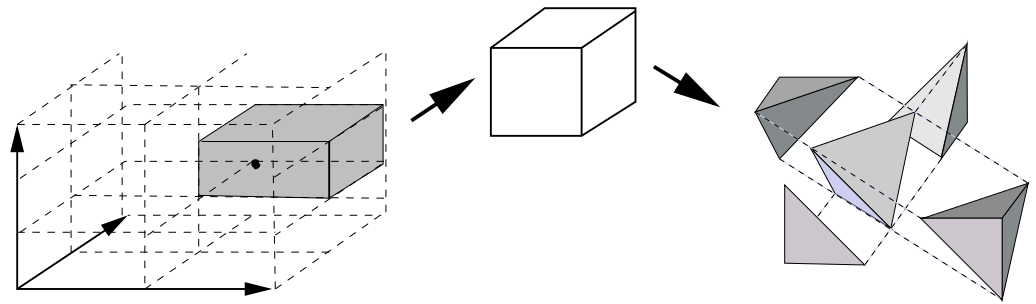

- Problem reduced to find the right simplex and $n+1$ function evaluations. 


\section{Interpolation Comparison}

\section{FINT-SIMP}

\section{Time Poli on a $1 \mathrm{GHz}$ P3}

\section{Interpolated value from 100 random vectors}

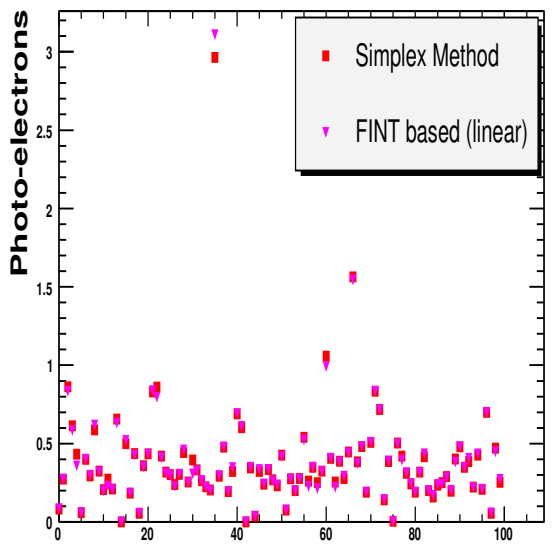

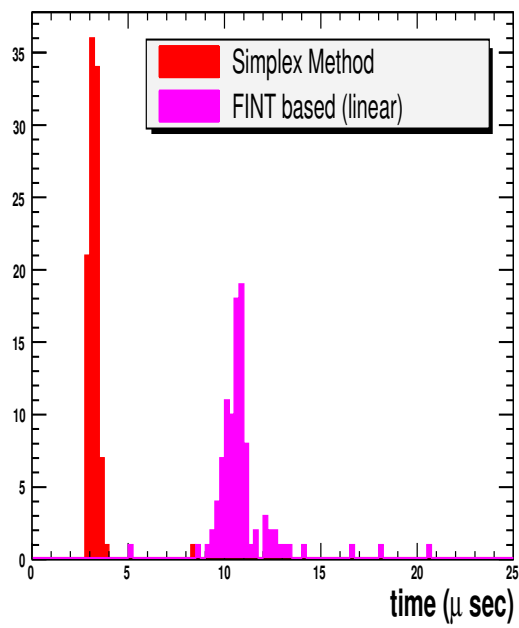




\section{Outline}

\section{Introduction}

\section{- The HERMES Experiment \\ - Design of the HERMES Lead-Glass Calorimeter}

\section{Simulation of the Lead Glass Calorimeter}

- Description of the Simulation

- Implementation of the Algorithm

(3) Results of the Simulation

- Different particles and observables 


\section{Electron initiated showers in the Calorimeter only}

\section{- Longitudinal Energy Deposition for perpendicular incidence -}

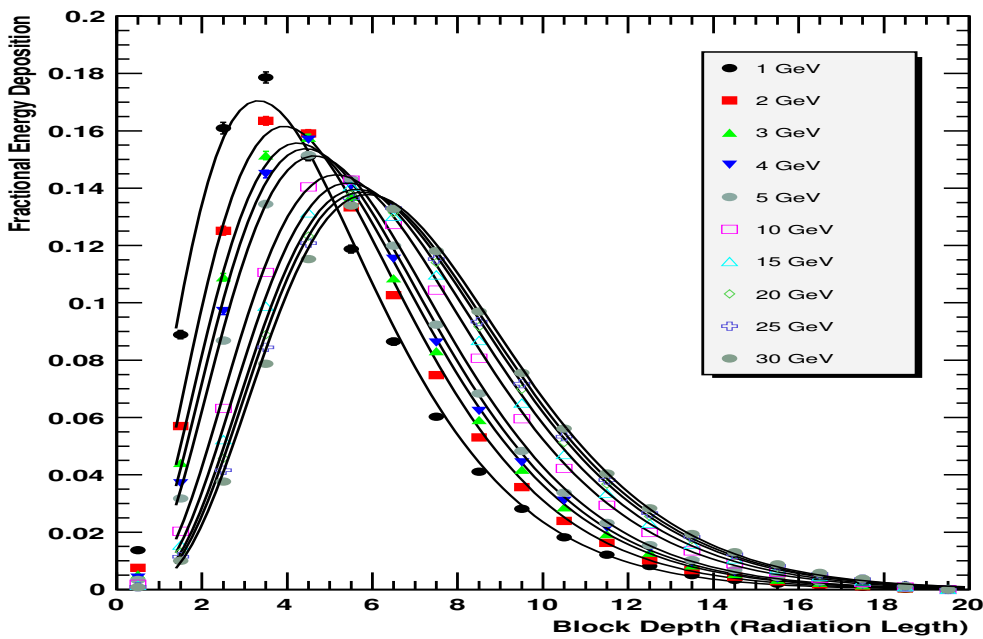




\section{Electron initiated showers in the Calorimeter only}

- Longitudinal Energy Deposition for perpendicular incidence -

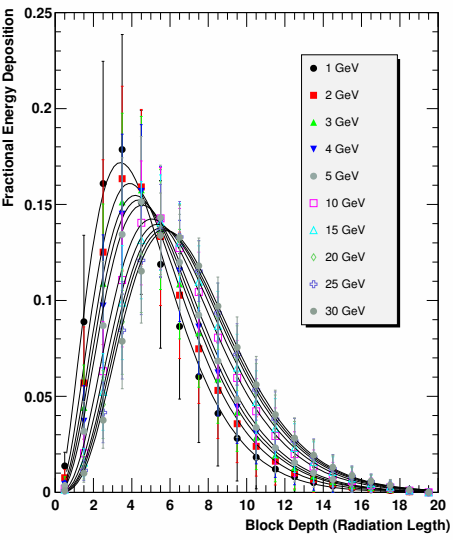

- Error bars = RMS of distribuition

- Resolution limited by shower fluctuations !

- NIM157 (1978) 455-460 reports an improved resolution when using a blue filter !
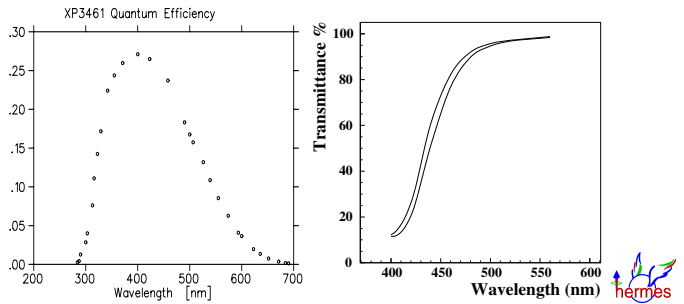


\section{Checking the interpolation}

- Generating $100 \mathrm{GeV}$ muons at different $(x, y)$ positions $\perp$ incidence -
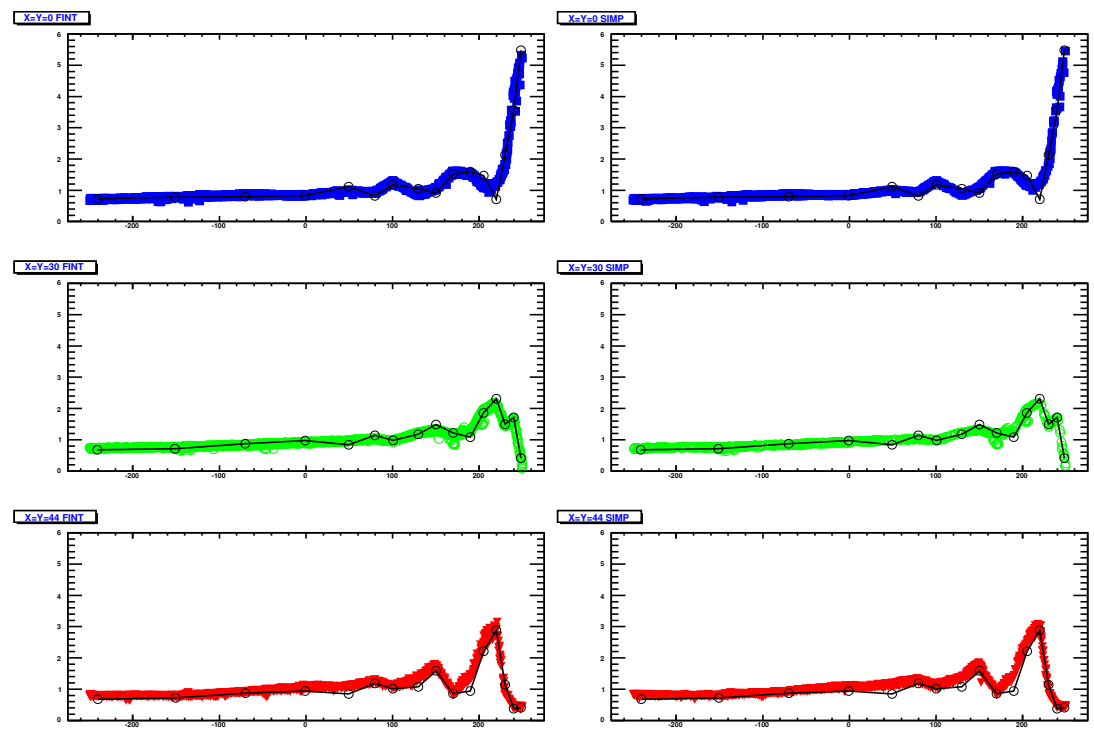


\section{Comparing to data: E/P leptons}

\section{- Generating $e^{-}$from the vertex -}

\section{simp_mean}

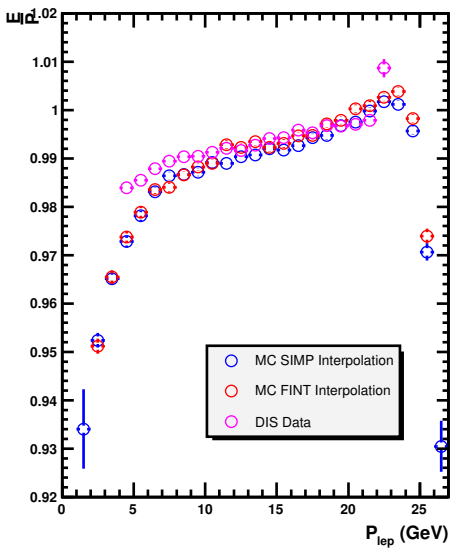

\section{simp_sigma}

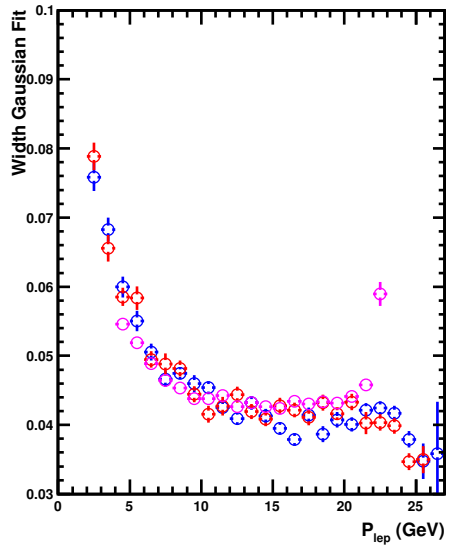

- $\mathrm{a} \sim 5 \%$ difference at $5 \mathrm{GeV}$ - Tracking in MC not perfect ! 


\section{Comparing to data: Photons}

\section{- Generating $\gamma$ from the vertex -}

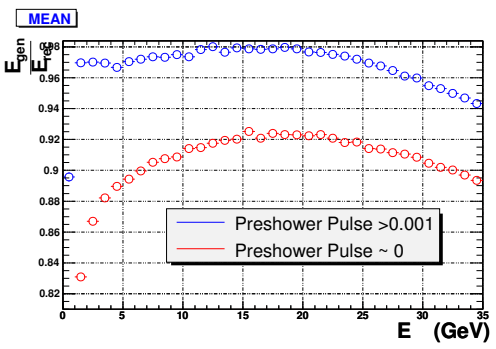

- No data to compare with! 


\section{Comparing to data: Photons}

\section{- Generating $\gamma$ from the vertex -}
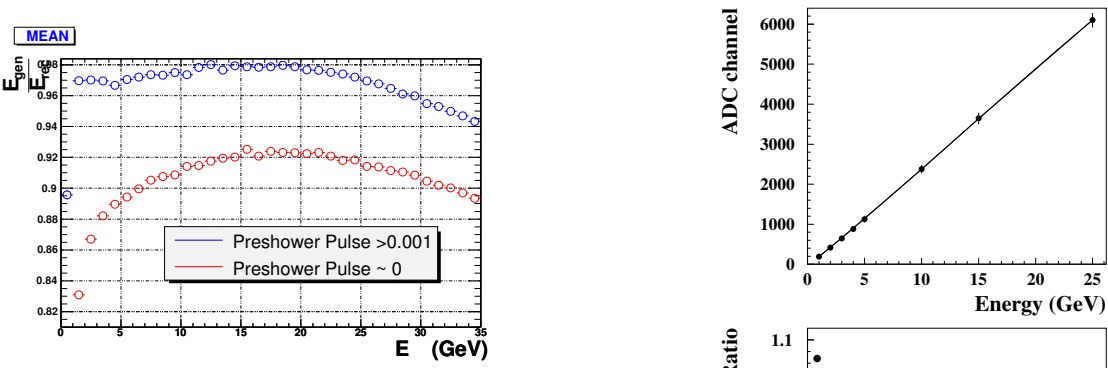

- No data to compare with!

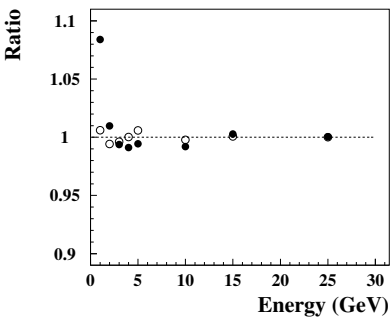

- Falloff due to cubic correction for the preshower !

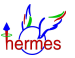




\section{Comparing to data: $\pi^{0}$}

- Blue: data, Red: MC -

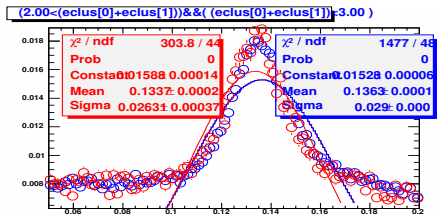

(3.00<(eclus[0]+eclus[1]))\&\&( (eclus[0]+eclus[1]) 4.00$)$

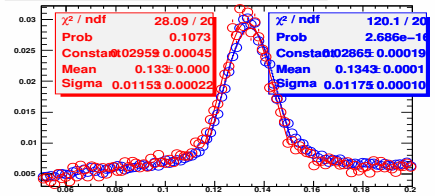

$(4.00<$ (eclus[0]+eclus[1]))\&\&( (eclus[0]+eclus[1]) 5.00$)$

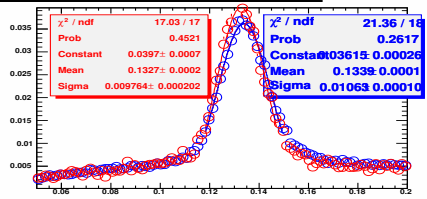

$\left(5.00<\right.$ (eclus[0]+eclus[1]))\&\&( (eclus[0]+eclus[1]) . $^{7.00)}$
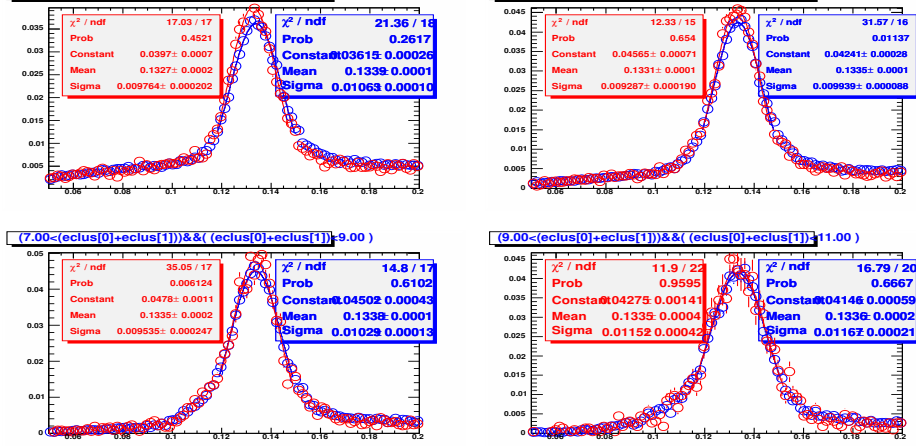

(9.00<(eclus[0]+eclus[1]))\&\&( (eclus[0]+eclus[1])-11.00)
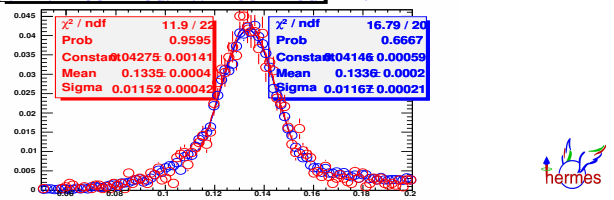

$\equiv \equiv \curvearrowleft \curvearrowright \curvearrowright$ 


\section{Comparison HMC/G3 - Standalone G4}

Not everything perfect !

- G4 and HMC/G3 simulation give a good overall agreement for muons

- For electrons HMC/G3 gets about $13 \%$ more photo-electrons than G4, while getting about $6 \%$ more Cherenkov Photons

been observed

- Total shower track length is different as well 


\section{Comparison HMC/G3 - Standalone G4}

Not everything perfect !

- G4 and HMC/G3 simulation give a good overall agreement for muons

- For electrons HMC/G3 gets about $13 \%$ more photo-electrons than G4, while getting about $6 \%$ more Cherenkov Photons

- A difference in escaping energy between G4 and HMC/G3 has been observed

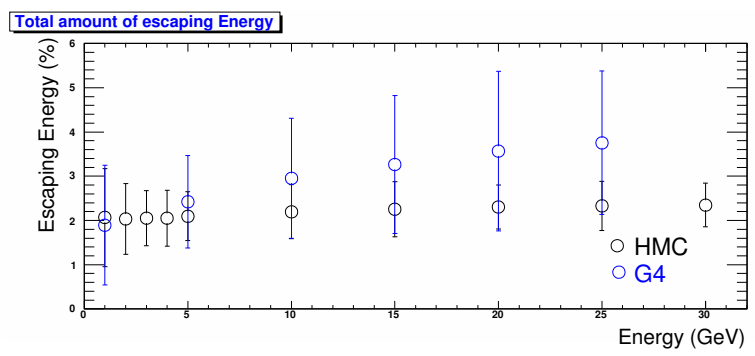




\section{Comparison HMC/G3 - Standalone G4}

Not everything perfect !

- G4 and HMC/G3 simulation give a good overall agreement for muons

- For electrons HMC/G3 gets about $13 \%$ more photo-electrons than G4, while getting about $6 \%$ more Cherenkov Photons

- A difference in escaping energy between G4 and HMC/G3 has been observed

- Total shower track length is different as well

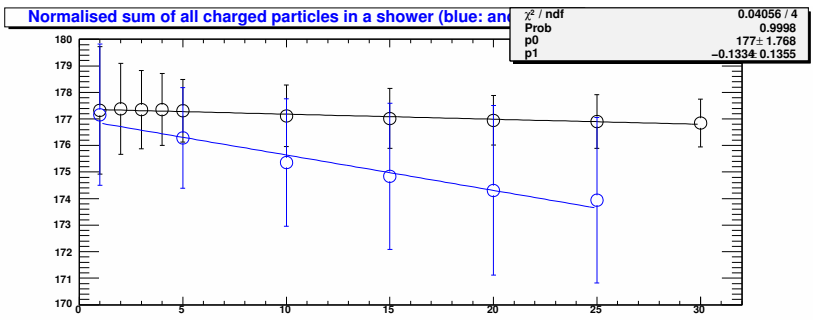




\section{Summary}

- The HeRmes electromagnetic calorimeter has been simulated using a 6 dimensional LUT generated by a standalone G4 MC

- Comparison with data shows a good agreement for E/P electrons and $\pi^{0}$ invariant mass spectrum

- Differences between G4 and HMC/G3 observed 


\section{For Further Reading I}

R. Ravotti et al.

'A Geometric Apporach to Maximum-Speed n-Dimensional Continuous Linear Interpolation in Rectangular Grids' IEEE Trans. on Comp. Vol 47 (1998), 894-899

H. Avakian et al.

'Performance of the electromagnetic calorimeter of the HERMES experiment'

NIM A417 (1998), 69-78 